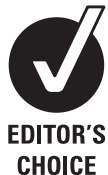

- Additional materials are published online only. To view these files please visit the journal online (http://jnnp.bmj. com)

${ }^{1}$ Department of Neurology, Leiden University Medical Centre, Leiden, The Netherlands ${ }^{2}$ Department of Neurology, Baylor College of Medicine, Houston, Texas, USA

\section{Correspondence to}

Dr $\mathrm{J} J$ van Hilten, Department of Neurology, Leiden University Medical Centre, P0 Box 9600, 2300 RC Leiden, The Netherlands; j.j.van hilten@lumc.nl

DEvR and EJG contributed equally to the manuscript.

Received 19 October 2010 Accepted 9 March 2011

Published Online First

14 April 2011

\title{
Peripheral trauma and movement disorders: a systematic review of reported cases
}

\author{
Diana E van Rooijen, ${ }^{1}$ Erica J Geraedts, ${ }^{1}$ Johan Marinus, ${ }^{1}$ Joseph Jankovic, ${ }^{2}$ \\ Jacobus J van Hilten ${ }^{1}$
}

\section{ABSTRACT}

Objective To perform a systematic review of cases reported in the literature in which a peripheral trauma preceded the onset of a movement disorder (MD). Methods Two reviewers independently searched Medline and EMBASE. Data regarding patient characteristics, type of MD and type of injury were collected, as well as information on the spread of MD, predisposing factors, psychological characteristics, presence of nerve lesions and treatment.

Results 133 publications presenting findings on 713 patients with peripherally induced movement disorders (PIMDs) were included. MDs were more frequent in women. The most commonly reported PIMD was fixed dystonia, which was often associated with pain and sensory abnormalities of the affected body part. In $26 \%$ of patients, a nerve injury was identified. More than one-third of patients had complex regional pain syndrome; these patients were younger, had a shorter interval before developing MDs and more often showed spread of MD to other body parts. Nearly $15 \%$ were diagnosed with a psychogenic movement disorder (PMD). PMD was associated with higher frequencies of fixed dystonia and tremor. In general, response to various treatments, including botulinum toxin administrations, was disappointing.

Conclusions While there is overlap in clinical characteristics between PIMDs and PMDs, the current review indicates that there are many well documented organic cases of PIMDs. This suggests that MDs, such as dystonia, tremor, myoclonus and tics, may under certain circumstances (eg, nerve lesions or genetic predisposition) be triggered by peripheral trauma. Potential mechanisms that may explain the underlying pathophysiology are addressed.

\section{INTRODUCTION}

Movement disorders (MDs) are a well recognised sequelae of brain injury secondary to head trauma ${ }^{1}$ but $\mathrm{MDs}$ associated with peripheral injury have not been well characterised. The first reports of such cases date back to 1888 when Gowers described two patients with abnormal involuntary movements that followed a local injury of the neck and thumb. ${ }^{2}$ Thereafter, this topic received little attention for almost a century. Over the last three decades, however, numerous reports on peripherally induced movement disorders (PIMDs) revived interest in this group of neurological disorders. ${ }^{3}$ The phenomenology of PIMDs often overlaps with otherwise typical dystonia, tremor, myoclonus, tics and other hyperkinetic and hypokinetic MDs of central origin. ${ }^{3-5}$
To facilitate research in this area, diagnostic criteria for PIMDs were developed ${ }^{5}$ and subsequently refined. $^{6}$ These criteria defined requirements with respect to the severity of the trauma, its anatomical relation to the site of the $\mathrm{MD}$ and to the interval between time of injury and onset of $\mathrm{MD}$. The criteria stimulated further research, but the pathophysiological mechanisms underlying PIMDs remained poorly understood and the topic has been a subject of some controversy. ${ }^{7-9}$ In order to explain the relatively rare occurrence of PIMDs compared with the frequency of peripheral injuries, it has been suggested that a peripheral trauma could trigger a PIMD in genetically or otherwise susceptible subjects with a pre-existent or subclinical MD. ${ }^{5} 10$ Another view suggested a predominantly psychogenic origin of PIMDs. ${ }^{11}$ The latter was based on several premises. Firstly, currently accepted theories on the pathogenesis of MDs have assumed a central origin of most MDs which could not be identified or supported in patients with PIMDs. Secondly, some characteristics related to onset, progression, phenotype and associated sensory problems in patients with PIMDs were different from those in patients with more typical $\mathrm{MDs}$ that arose without preceding peripheral trauma. Finally, some patients with PIMDs had coexistent psychological problems, a history of psychiatric disease or other clinical features that supported the 'psychogenic' basis of PIMDs.

One of the difficulties in studying PIMDs is their relative rarity, which impedes the application of epidemiological studies to this group of MDs. Cohort studies may not be appropriate if diseases are uncommon whereas case control studies are only applicable for identifying frequent risk factors. Another challenge is the heterogeneous nature of PIMDs and the lack of large, well characterised series. Against this background, we conducted a systematic review of the characteristics of published PIMD cases. Additionally, mechanisms underpinning potential causality of peripheral trauma in the development of MDs are discussed.

\section{METHODS}

\section{Search strategy}

A literature search was performed in Medline and EMBASE in order to identify potentially relevant studies. The search strategy was designed together with a specialist in information retrieval methods of the Leiden University Medical Centre library, and comprised a combination of controlled vocabulary (MeSH terms) as well as free text terms related to peripheral injuries combined with (types of) 
movement disorders (see supplement 1, figure 1, available online only). The results were limited to articles in English, German and Dutch. The most recent search was performed on 31 December 2009.

\section{Selection criteria}

The eligibility of the reports, as identified by the search strategy, was independently assessed by two authors (DvR, EG). This assessment was not blind with respect to authors or institution. The identified studies were first screened by title and abstract, after which the full text of potential relevant articles was studied. Reports were only included if the onset of the MD was preceded by a peripheral trauma. We defined peripheral trauma as a tissue or peripheral nerve injury (NI) evoked by an external physical stimulus (ie, mechanical, electrical, thermal or radiation). The $\mathrm{MDs}$ were required to have an onset at or in close proximity to the anatomical site of the precipitating trauma. ${ }^{5} 6$ We set no limits on the severity of the injury because it is known that even mild trauma may evoke an $\mathrm{MD}$, or on the duration of the interval between injury and onset of the $\mathrm{MD}$ because theoretical background for any cut-off is lacking. ${ }^{12}$ Patients with hemifacial spasm, an $\mathrm{MD}$ with an accepted peripheral causative origin, were excluded. Additionally, in order to rule out the possibility of CNS pathology as a cause of MDs, cases with head trauma or spinal cord injury were excluded. Other exclusion criteria were primary dystonia (including task related dystonia) and prior neuroleptic use. Additionally, reference lists of all included publications as well as reviews on this topic were tracked following the same review procedure as described above. Discrepancies between the reviewers were resolved by a consensus agreement.

\section{Data extraction}

Data regarding patient characteristics, type of $\mathrm{MD}$, type of trauma preceding the onset of the $\mathrm{MD}$, potential spread of the $\mathrm{MD}$, potential predisposing (psychogenic and non-psychogenic) factors, psychological characteristics, other signs and symptoms (eg, sensory and autonomic changes), nerve lesions and treatment was extracted using a standard form. Neurophysiological evidence indicative of peripheral nerve lesions included: slow nerve conduction, fasciculations, sharp waves and fibrillations. In addition, nerve specific deficits on examination, as well as nerve compression revealed by imaging studies or detected during surgery, were considered as NI.

No quality assessment for each report was performed since, to the best of our knowledge, no appropriate scales exist for this type of research.

\section{Statistical analysis}

Standard descriptive statistics were calculated using SPSS (V.17.0) - that is, means or medians (as appropriate) and percentages. For studies in which only group means were described for certain characteristics, weighted means (with the number of involved patients used as weight) were calculated and used for further analysis. Differences between groups were assessed with Mann-Whitney $U$ tests and $\chi^{2}$ tests. A $p$ value $<0.05$ was considered statistically significant.

\section{RESULTS}

\section{Preceding events and patient characteristics}

A total of 47 full reports and 86 case reports met the criteria for inclusion (see supplement 1, figure 2; and supplement 2, available online only). Together, these reports included data from 713
Table 1 Peripherally induced movement disorder characteristics

\begin{tabular}{|c|c|}
\hline Total No of patients (\% females) & $713(64)$ \\
\hline Age at onset of movement disorder (years) (median (IQR)) $(n=571)$ & $38(30-53)$ \\
\hline No of patients with CRPS & $260(36)$ \\
\hline No of patients with PMD & $103(14)$ \\
\hline \multicolumn{2}{|l|}{ Trauma (n (\%)) } \\
\hline Soft tissue injury & $310(43)$ \\
\hline Fracture & $71(10)$ \\
\hline Surgery & $74(10)$ \\
\hline Other & $87(12)$ \\
\hline Nerve entrapment & $130(18)$ \\
\hline Amputation & $12(2)$ \\
\hline Not described & $29(4)$ \\
\hline Time to onset of movement disorder (days) (median (IQR)) $(n=264)$ & $21(2-183)$ \\
\hline \multicolumn{2}{|l|}{ Type of movement disorder (n (\%)) } \\
\hline Dystonia & $513(72)$ \\
\hline \multicolumn{2}{|l|}{ Type of dystonia } \\
\hline Fixed (\% of dystonia cases) & $317(62)$ \\
\hline Mobile & $102(20)$ \\
\hline Both & $56(11)$ \\
\hline Not specified & $38(8)$ \\
\hline \multicolumn{2}{|l|}{ Sensory trick } \\
\hline Yes/no & 29/110 \\
\hline Tremor & $176(25)$ \\
\hline Myoclonus & $95(13)$ \\
\hline Spasms & $79(11)$ \\
\hline PLMT/F & $46(6)$ \\
\hline Other (chorea, parkinsonism, tics) & $26(4)$ \\
\hline \multicolumn{2}{|l|}{ Location (n (\%)) } \\
\hline Face & $1(0)$ \\
\hline Oromandibular/vocal cords & $44(6)$ \\
\hline Neck/shoulder & $176(25)$ \\
\hline Trunk & $14(2)$ \\
\hline Arm & $162(23)$ \\
\hline Leg & $177(25)$ \\
\hline Unknown extremity & $125(18)$ \\
\hline Multiple sites & $14(2)$ \\
\hline \multicolumn{2}{|l|}{ Spread of movement disorder to other body regions $(n(\%))^{*}(n=138)$} \\
\hline Ipsilateral & $15(11)$ \\
\hline Contralateral & $16(12)$ \\
\hline Segmental & $14(10)$ \\
\hline Generalised & $35(25)$ \\
\hline Multifocal & $51(37)$ \\
\hline Unknown & 7 (5) \\
\hline
\end{tabular}

$\mathrm{n}$, indicates the number of patients on which this information was available; (\%) refers to the whole group unless otherwise specified.

* Percentage calculated for the number of patients on which the information was available. CRPS, complex regional pain syndrome; PLMT/F, painful limbs and moving toes or fingers; PMD, psychogenic movement disorder.

PIMD cases. Most MDs occurred after soft tissue injuries (43\%), followed by fractures (10\%) and surgery $(10 \%)$ (table 1$)$. Median age of the cases was 38 years (IOR 30-53) and the majority involved women (64\%).

\section{Movement disorders and locations}

The most frequently reported $\mathrm{MD}$ was dystonia (72\%). In cases where the type of dystonia was not specifically labelled (94\%), we categorised the type of dystonia as 'mobile' if dystonic movements were reported (20\%) and 'fixed' if the $\mathrm{MD}$ was described as a 'fixed', 'tonic' or 'dystonic' posture (62\%). The remainder had either a mixed type (11\%) or the type of dystonia was not specified (8\%). The presence or absence of sensory tricks was described in $27 \%$ of all dystonia patients and were effective in $6 \%(5 \%(4 / 80)$ of patients with fixed vs $33 \%(14 / 42)$ of patients with mobile dystonia). Other reported $\mathrm{MDs}$ were 
tremor $(25 \%)$, myoclonus $(13 \%)$, spasms (11\%) and painful limbs moving toes or fingers (6\%) while another $4 \%$ had parkinsonism, chorea and tics (table 1). The total number of MDs exceeded $100 \%$ because of the coexistence of different MDs in some patients (160 cases, two MDs; 62 cases, three MDs).

In $66 \%$ of the cases the $\mathrm{MD}$ started in a limb, in $25 \%$ in the neck and shoulder region, in $2 \%$ in the truncal region and in $6 \%$ in the oromandibular region. In $2 \%$, the $\mathrm{MDs}$ started simultaneously in multiple regions. Spreading of $\mathrm{MD}$ from the original site to other body parts was described in 19\% of all cases.

\section{Time interval}

The interval between peripheral trauma and onset of MDs showed a large variability across studies, with most studies $(95 \%)$ reporting latencies of less than 1 year. Median time interval was 21 days (IOR 2-183 days). In 29 cases (4\%), the reported interval was more than 1 year; the clinical characteristics of these cases did not differ from the cases that developed the PIMD within 1 year.

\section{Nerve injury}

Overall, 182 patients (26\%) showed evidence of an NI (table 2) which was demonstrated by neurophysiological examination $(7 \%)$, imaging studies (2\%), neurological examination $(3 \%)$ or revealed by an operation (3\%). In another $10 \%$ of patients, the presence of nerve injury was reported although the means by which this was established was not specified.

\section{Pain and other sensory disturbances}

Pain was reported in the majority of patients (86\%) and preceded the onset of $\mathrm{MD}$ in $19 \%$ of cases. Sensory impairments of the affected body parts were noted in $42 \%$ of all patients. Positive (ie, hyperalgesia, allodynia) and negative (ie, hypoalgesia, hypoesthesia) sensory symptoms were equally distributed. Complex regional pain syndrome (CRPS) was diagnosed in $36 \%$ of patients and their characteristics were compared with those of non-CRPS patients (see supplement 3 , table 4 , available online only). Only 8\% of the CRPS patients had an NI (CRPS type II) compared with $36 \%$ in the non-CRPS group. The proportion of females was higher in CRPS patients than in non-CRPS patients ( $80 \%$ vs $55 \%$ ). CRPS and non-CRPS patients differed on types of trauma and locations of the MD. Dystonia was the most frequently reported $\mathrm{MD}$ in both groups and was almost always of a fixed type in CRPS patients (91\%) while a more equal distribution of fixed and mobile type was found in non-CRPS patients. Spread of MDs was more frequently reported for CRPS patients compared with non-CRPS patients (34\% vs 11\%).

\section{Psychogenic movement disorders}

Fourteen percent of the patients were diagnosed with a psychogenic movement disorder (PMD). The other $86 \%$ $(n=610)$ of PIMD cases were considered non-psychogenic, either because the original authors explicitly stated this or because potential psychogenic causes were not discussed. In 59\% of the PMD patients the diagnostic criteria for documented or clinical established PMD were fulfilled. The most common psychiatric diagnosis was a somatisation disorder (7\%) or conversion disorder $(18 \%)$, while in $18 \%$ a psychiatric assessment could not establish a diagnosis. Other features that were frequently documented in this group were abrupt onset (47\%), inconsistency over time $(12 \%)$, multiple somatisations $(12 \%)$, false neurological signs (31\%) and distractibility (28\%).

Cases with and without PMDs are compared in table 3 . In cases without PMD, psychological features were described in
Table 2 Additional information on peripherally induced movement disorders

\begin{tabular}{|c|c|}
\hline \multicolumn{2}{|l|}{ Nerve lesion identified by $(\mathrm{n}(\%))^{*}(\mathrm{n}=182)$} \\
\hline Electrophysiology & $50(27)$ \\
\hline Imaging & $17(9)$ \\
\hline Neurological examination & $21(12)$ \\
\hline Operation & $24(13)$ \\
\hline Unspecified & $70(38)$ \\
\hline \multicolumn{2}{|l|}{ Pain $(n(\%))^{*}(n=568)$} \\
\hline Before start of movement disorder & $132(23)$ \\
\hline Simultaneous with start of movement disorder & $225(40)$ \\
\hline Present, but time unknown & $186(33)$ \\
\hline Not present & $25(4)$ \\
\hline \multicolumn{2}{|l|}{ Sensory signs $(\mathrm{n}(\%))^{*}(\mathrm{n}=301)$} \\
\hline Hypo (hypalgesia) & $94(31)$ \\
\hline Hyper (allodynia, hyperalgesia) & $83(28)$ \\
\hline Both & $45(15)$ \\
\hline Altered sensation, type deficit not described & $79(26)$ \\
\hline \multicolumn{2}{|l|}{ Psychological aspects $(n(\%))^{*}(n=258)$} \\
\hline Present & $84(33)$ \\
\hline Not present & $174(67)$ \\
\hline \multicolumn{2}{|l|}{ Litigation $(\mathrm{n}(\%))^{*}(\mathrm{n}=200)$} \\
\hline Involved in litigation/worker's compensation & $66(33)$ \\
\hline Not involved in litigation & 134 (67) \\
\hline \multicolumn{2}{|l|}{ Treatment with BoNT $(n(\%))^{*}(n=133)$} \\
\hline Successful & $26(20)$ \\
\hline Partly successful & $75(56)$ \\
\hline Passive successful & $3(2)$ \\
\hline Unsuccessful & $29(22)$ \\
\hline \multicolumn{2}{|l|}{ Predisposing factors $(\mathrm{n}(\%))^{*}(\mathrm{n}=265)$} \\
\hline Family history of similar movement disorder & $15(6)$ \\
\hline Family history of other movement disorder & $6(2)$ \\
\hline Drug abuse & $1(0)$ \\
\hline Premature birth & $1(0)$ \\
\hline Developmental delay & $1(0)$ \\
\hline Other & $27(10)$ \\
\hline No predisposing factors & $214(81)$ \\
\hline
\end{tabular}

$30 \%$ of all cases but only formally evaluated by a psychologist or psychiatrist in $9 \%$. Twenty-seven of these patients (4\%) showed symptoms of depression and anxiety associated with onset of the MD while two patients had a medical history of anxiety or psychosis. Patients with PMD more often had fixed dystonia ( $90 \%$ vs $58 \%$ ) and tremor (38\% vs $22 \%$ ), and less often mobile dystonia ( $6 \%$ vs $22 \%$ ), myoclonus ( $6 \%$ vs $15 \%$ ), painful limbs and moving toes or fingers ( $0 \%$ vs $7 \%$ ) or miscellaneous MDs (10\% vs 3\%). There were differences between PMD and non-PMD cases in the distribution of affected body regions and eliciting events. Patients with PMD were also more involved in lawsuits or received worker's compensation (notably, information on these topics was more often available for PMD patients, $63 \%$ vs $22 \%$ ).

\section{Predisposing factors}

Potential predisposing factors of MDs were addressed in 265 cases and identified in 51 (19\%). A family history of an $\mathrm{MD}$, similar to the one that occurred in the index case (although not peripherally induced or in the same region), was described in 15 patients. In 27 cases it was not possible to exclude other factors that may have contributed to the onset of the PIMDs (eg, HIV, previous peripheral trauma or radiotherapy). In two cases, more than two predisposing factors were reported. 
Table 3 Characteristics of patients with and without psychogenic movement disorder*

\begin{tabular}{|c|c|c|c|}
\hline & PMD & Without PMD & p Value \\
\hline Total No of patients ( $\mathrm{n}(\%$ females)) & $103(61)$ & $610(64)$ & 0.59 \\
\hline Age at onset (years) (median (IOR)) & $37(28-47) n=102$ & $39(31-54) n=469$ & 0.03 \\
\hline No of patients with CRPS (\%) & $10(10)$ & $250(41)$ & $<0.001$ \\
\hline Trauma (n (\%)) & & & $<0.001 \S$ \\
\hline Soft tissue injury & $45(44)$ & $265(43)$ & \\
\hline Fracture & $5(5)$ & $66(11)$ & \\
\hline Surgery & $14(14)$ & $60(10)$ & \\
\hline Amputation & $1(1)$ & $11(2)$ & \\
\hline Nerve entrapment & $1(1)$ & $129(21)$ & \\
\hline Other & $37(36)$ & $50(8)$ & \\
\hline Not described & & $29(5)$ & \\
\hline Time to onset (days) (median (IQR)) & $14(7-61)$ & $21(2-183)$ & 0.54 \\
\hline \multicolumn{4}{|l|}{ Type of movement disorder (n (\%)) } \\
\hline Dystonia & $67(65)$ & $446(73)$ & $0.09 \S$ \\
\hline \multicolumn{4}{|l|}{ Type of dystonia (\% of dystonia cases) } \\
\hline Fixed & $60(90)$ & $257(58)$ & $<0.001$ \\
\hline Mobile & $4(6)$ & $98(22)$ & \\
\hline Both & $1(1)$ & $55(12)$ & \\
\hline Unknown & $2(3)$ & $36(8)$ & \\
\hline Tremor & $39(38)$ & $137(22)$ & $<0.001$ \\
\hline Myoclonus & $6(6)$ & $89(15)$ & 0.015 \\
\hline Spasms & $15(15)$ & $64(10)$ & 0.22 \\
\hline $\mathrm{PLMT} / \mathrm{F}$ & - & $42(7)$ & $<0.001$ \\
\hline Other (chorea, parkinsonism, tics) & $10(10)$ & $16(3)$ & $<0.001$ \\
\hline Spread of movement disorder to other body regions (n (\%)) & $33(32)$ & $118(19)$ & 0.004 \\
\hline Location (n (\%)) & & & $<0.001 \S$ \\
\hline Face & - & $1(0)$ & \\
\hline Oromandibular/vocal cords & - & $44(7)$ & \\
\hline Neck/shoulder & $20(19)$ & $156(26)$ & \\
\hline Trunk & - & $14(2)$ & \\
\hline Arm & $40(39)$ & $122(20)$ & \\
\hline Leg & $34(33)$ & $143(23)$ & \\
\hline Extremity not specified & - & $125(21)$ & \\
\hline Multiple sites & $9(9)$ & $5(1)$ & \\
\hline \multicolumn{4}{|l|}{ PMD criteria† $(\mathrm{n}(\%)) \ddagger$} \\
\hline Documented/clinically established & $61(76)$ & - & \\
\hline Probable/possible & $16(20)$ & - & \\
\hline Not fulfilled & $3(4)$ & - & \\
\hline \multicolumn{4}{|l|}{ Psychiatric diagnosis $†(n(\%)) \ddagger$} \\
\hline Conversion/somatisation disorder & $26(35)$ & - & \\
\hline Multiple diagnoses & 7 (9) & - & \\
\hline Malingering & $2(3)$ & - & \\
\hline Other & $20(27)$ & $10(5)$ & \\
\hline No psychiatric diagnosis established & $19(26)$ & $54(29)$ & \\
\hline Clinical psychological features (eg, depression) & - & $19(10)$ & \\
\hline No clinical psychological features & - & $101(59)$ & \\
\hline Litigation/worker's compensation† $(\mathrm{n}(\%)) \neq$ & $39(60)$ & $27(20)$ & $<0.001$ \\
\hline Nerve lesion† $(\mathrm{n}(\%)) \ddagger$ & $2(50)$ & $180(68)$ & $<0.001$ \\
\hline \multicolumn{4}{|l|}{ Pain† $(\mathrm{n}(\%)) \neq$} \\
\hline Present & $50(98)$ & $493(95)$ & $<0.001$ \\
\hline
\end{tabular}

$\mathrm{n}$, number of patients on which this information was available; (\%) refers to whole group unless otherwise specified.

*Differences between groups should be interpreted with caution: if variables were not described it was assumed that these variables were not present. This could lead to distortion of the $p$ values, especially in features reported in less than $50 \%$ of the cases (indicated by $\dagger$ ).

¡Percentage calculated for the number of patients on which the information was available.

$\S p$ values indicate omnibus tests; $p$ values without a symbol are comparisons of one variable.

CRPS, complex regional pain syndrome; PLMT/F, painful limbs and moving toes or fingers; PMD, psychogenic movement disorder.

\section{Treatment}

The applied treatments differed greatly across studies and included oral pharmacotherapy (anticholinergics, baclofen, benzodiazepines, levodopa), botulinum toxin (BoNT), physical therapy, deep brain stimulation and nerve decompression surgery. BoNT was the most frequently applied therapy (19\%) but was found to be successful in only $20 \%$ of these cases (table 2). There were two reports describing single CRPS patients who underwent brain surgery. One patient with fixed dystonia was treated with thalamic and pallidal deep brain 
stimulation without any improvement of the $\mathrm{MD}$ or pain. In the other patient, stereotaxic thalatomy abolished the muscle spasms and reduced the pain.

\section{DISCUSSION \\ Clinical features}

In this systematic review, we identified 133 studies that recorded information on 713 cases with PIMDs. Our findings show that both the nature and severity of a peripheral trauma preceding the onset of an $\mathrm{MD}$ may vary considerably. In almost half of the patients, the occurrence of an $\mathrm{MD}$ was preceded by soft tissue trauma, but in other cases, various types of trauma, such as surgery, were reported. The median time interval between peripheral trauma and the onset of $\mathrm{MD}$ was 21 days. In order to explain the obvious discrepancy between high frequency of peripheral trauma and very low occurrence of PIMDs, it has been suggested that genetic susceptibility and other predisposing factors may play a role. ${ }^{5} 13$ In one series, peripheral trauma played a role in idiopathic torsion dystonia in $16 \%$ of the cases. ${ }^{10}$ In a case control study, neck or trunk trauma was found to be associated with an increased risk of developing cervical dystonia while ocular diseases increased the risk of developing blepharospasm. ${ }^{14}$ In most cases of dystonia, such as writer's cramp ${ }^{15}$ and DYT1 dystonia, ${ }^{16-18}$ however, no prior peripheral trauma had been documented. In contrast, trauma is commonly reported prior to the onset of DYT12 dystonia, also known as rapid onset dystonia parkinsonism. ${ }^{19}$ Although a confounding influence of recall bias cannot be completely ruled out in some of the reported cases of PIMDs, our thorough review of the literature suggests that peripheral trauma indeed may play a role in triggering the onset of an $\mathrm{MD}$ or in exacerbating pre-existing idiopathic or genetic dystonias.

Dystonia was the most frequent PIMD encountered in this review, with fixed dystonia being more common than mobile dystonia. Although peripherally induced dystonia may be phenomenologically indistinguishable from idiopathic dystonia, the former is more commonly manifested by fixed dystonia. ${ }^{13}$ More than one-third of patients with PIMDs were also diagnosed with CRPS. Compared with dystonia patients without CRPS, those with associated CRPS were usually women, younger at onset and more often showed a spread of symptoms. Only $8 \%$ of patients with CRPS had an identified NI and, by definition, thus actually suffered CRPS type II. When fixed dystonia is associated with CRPS it generally has a poor prognosis. ${ }^{20}$ In the absence of a clear and generally accepted pathophysiological or anatomical substrate for a disease, nonorganic explanations, including psychological causes, are often advanced as potential mechanisms. ${ }^{11}$ The criteria for diagnosing PMD were first described by Fahn and Williams (1988) and classified as documented, clinically established, probable and possible PMD. The distinction between PIMDs and PMDs is complicated as about a third of PMDs are also preceded by a physical injury. ${ }^{21}$ Furthermore, a psychiatric diagnosis is absent in one-third of cases with PMD, and PMDs may have a similar phenotype as PIMDs. ${ }^{11} 22$ This especially holds for fixed dystonia, ${ }^{23}$ a finding that is confirmed by the results of this review. Pending litigation or secondary gain has been found to be associated with PMDs although there is no evidence that the outcome of litigation influences the prognosis of the MD. ${ }^{6} 24$ The results show that PMD patients were more involved in lawsuits and worker's compensation than patients without this diagnosis. However, it is difficult to draw firm conclusions from comparisons between PMDs and non-PMDs as the description of features may largely depend on the researcher's perspective.

Research on the topic of PIMDs is also hampered by the lack of objective physiological or other markers that can reliably discriminate between MDs of organic and psychogenic origin. ${ }^{25}$ Nuclear imaging and neurophysiological investigations may be helpful in detecting psychogenic parkinsonism, tremor and myoclonus. ${ }^{26-28}$ Transcranial magnetic stimulation was used to evaluate patients with organic and psychogenic dystonia. ${ }^{26}$ This method showed similar short interval cortical inhibition in patients with organic and fixed dystonia ${ }^{29}$ and in patients with organic and psychogenic dystonia. ${ }^{30}$ However, patients with organic dystonia exhibited abnormal motor cortical plasticity measured by a paired associative stimulation protocol compared with patients with psychogenic dystonia. ${ }^{31}$ Notably, there is still some debate regarding the methodology and the interpretation of the results emerging from this paradigm. ${ }^{32} 33$

\section{Possible pathogenesis of PIMDs}

Studies of the pathophysiology of hemifacial spasm have generated hypotheses for the mechanisms of other PIMDs. Vascular compression of the facial nerve at its root exit zone from the brainstem is demonstrated in most cases, and microvascular decompression is usually effective in relieving this $\mathrm{MD} .{ }^{34} \mathrm{~A}$ few cases of torticollis associated with neurovascular compression of the accessory nerve have been reported with remission of symptoms after decompression surgery. Further studies are needed to better understand the possible relationship between torticollis and this form of 'peripheral injury'.

Given the fact that PIMDs are usually preceded by peripheral tissue damage or NI (present in approximately $30 \%$ of all patients) and are commonly associated with pain (80\%) and sensory abnormalities of the affected part of the body (37-50\%), we postulate that the initial insult interferes with normal sensory processing, which eventually leads to spinal or supraspinal reorganisation.

There is a growing body of evidence from animal models that experimentally induced peripheral NI is associated with cell death of axotomised neurons as well as prominent manifestations of neuroplasticity, including reorganisation of afferent projections, involvement of glia cells ${ }^{35}$ and changes in synaptic efficacy. ${ }^{36}$ These latter changes have been studied mainly in the context of chronification of pain; little is known about their role in the development of MDs. Interestingly, a rat model of neuropathic pain showed clawed postures after lesions of the sciatic and tibial nerve. The prevalence of postural abnormalities that resemble dystonia in these rats correlated with the degree of nerve damage. However, contrary to the situation in humans, the majority of these postures disappeared within 2 weeks. ${ }^{37}$

Information on the presence or absence of NI was available in less than half of the patients in our review. One-third were diagnosed with CRPS type I and may have had pathology of the small diameter afferents, which goes unnoticed on clinical examination or standard electrophysiological testing. ${ }^{38}$ However, the role of small diameter afferents in the pathogenesis of dystonia is uncertain as CRPS patients with and without dystonia show similar reduced cold and warmth detection thresholds. ${ }^{39}$

In CRPS, central sensitisation of spinal neurons underpins the occurrence of allodynia, chronification of pain and spread of pain hypersensitivity beyond the area of tissue damage. ${ }^{3640}$ Although research on central sensitisation has hitherto focused on sensory features, animal models show that this process may also influence spinally mediated motor behaviours such as enhancement 
of nociceptive withdrawal reflexes. ${ }^{41}$ Furthermore, central sensitisation is associated with loss of inhibition along the neuraxis, as demonstrated in CRPS patients with dystonia. ${ }^{42} 43$ Dystonia in CRPS patients may respond to the $\mathrm{GABA}_{\mathrm{B}}$ agonist baclofen, which enhances spinal GABAergic inhibition. ${ }^{41} 44$ Baclofen specifically stimulates $G A B A_{B}$ receptors, which inhibit sensory input on neurons of the spinal cord.

In primary dystonia, abnormalities of higher order sensory processes, including sensory temporal-spatial discrimination, multisensory integration and movement representation, are common and are assumed to be related to dysfunction of the basal ganglia cortico-striatal-thalamo-cortical motor circuits. ${ }^{45}$ Cortical reorganisation may occur after changes in sensory input $^{36}$ where maladaptive changes may lead to the development of dystonia in susceptible individuals. ${ }^{46}$ Collectively, the available evidence suggests that peripherally initiated conditions may change anatomical and functional connectivity of spinal and supraspinal sensorimotor circuits, which in turn may lead to chronification of pain, sensory impairments and abnormal centrally mediated motor responses.

\section{Treatment of PIMDs}

Responses to various treatment interventions are generally disappointing in PIMD patients. BoNT was the most commonly used treatment, administered in one-fifth of all patients. Contrary to the marked beneficial effect of BoNT in typical focal or segmental dystonia, application of this treatment in patients with PIMDs was found to be satisfactory in only $20 \%$, which corroborates with previous observations. ${ }^{47}$ The poor response to BoNT can be due to the fixed nature of dystonia, as the development of contractures may limit the usefulness of BoNT. Another explanation may lie in the fact that it is generally more difficult to obtain a satisfactory response in PIMD, as they mainly occur in (particularly distal) limbs, with often many muscles involved in the dystonic posturing. As these patients represent primary nonresponders, immunoresistance due to blocking antibodies, detected in less than $2 \%$ of patients repeatedly treated with BoNT, is an unlikely explanation for the lack of response. ${ }^{48}$

There are several limitations of our review that must be acknowledged. Firstly, even though two authors independently searched the literature, potential relevant articles may have been missed. Secondly, as PIMD is a controversial issue, underreporting may have occurred. Moreover, the quality of reported data differed considerably between studies, with some articles providing insufficient information on the characteristics of interest (or were not evaluated for all features) and the data are subject to recall bias. As prospective studies are very difficult to perform in this type of disorder, it is important that future studies report detailed information on all characteristics related to the $\mathrm{MD}$, the results of additional neurophysiological examinations, psychological or psychiatric assessment, potential predisposing factors and the long term outcomes.

In conclusion, based on our review of more than 700 cases of PIMDs reported in 133 articles, we show that psychological factors may have played a role in a proportion of the patients. However, in a substantial number of patients in whom psychological features were not present or could not be identified, peripheral trauma indeed may have triggered a central process which underpins the subsequent onset of MDs. Objective tools are needed to improve sensitivity and specificity for the diagnosis of PMDs in order to optimise treatment strategies. We postulate that aberrant peripheral input, possibly in combination with genetic and other predispositions, leads to disturbed connectivity in spinal and supraspinal sensorimotor circuits associated with central reorganisation and subsequent development of $\mathrm{MDs}$. The poor response to BoNT and other treatment modalities in patients with PIMD argues for future research to further explore the potential mechanisms of PIMD so that effective, pathogenesis targeted therapies can be eventually developed.

Acknowledgements The authors thank Jessica Langenhof and Jan Schoones of the Waleus Library for their help with the search strategy.

Funding This study is part of TREND (Trauma RElated Neuronal Dysfunction), a Dutch Consortium that integrates research on epidemiology, assessment technology, pharmacotherapeutics, biomarkers and genetics on complex regional pain syndrome type 1. The consortium aims to develop concepts on disease mechanisms that occur in response to tissue injury, its assessment and treatment. TREND is supported by an unrestricted grant from the Dutch Ministry of Economic Affairs (BSIK03016).

\section{Competing interests None.}

Provenance and peer review Not commissioned; externally peer reviewed.

\section{REFERENCES}

1. Krauss JK, Jankovic J. Head injury and posttraumatic movement disorders. Neurosurgery 2002;50:927-39.

2. Gowers WR. A manual of diseases of the nervous system. London: Churchill, 1888

3. Jankovic J. Peripherally induced movement disorders. Neurol Clin 2009;27:821-32.

4. Goetz CG, Pappert EJ. Trauma and movement disorders. Neurol Clin 1992;10:907-19.

5. Jankovic J, Van der LC. Dystonia and tremor induced by peripheral trauma: predisposing factors. J Neurol Neurosurg Psychiatry 1988;51:1512-19.

6. Cardoso F, Jankovic J. Peripherally induced tremor and parkinsonism. Arch Neurol 1995; 52:263-70

7. Jankovic J. Can peripheral trauma induce dystonia and other movement disorders? Yes! Mov Disord 2001;16:7-12.

8. Weiner WJ. Can peripheral trauma induce dystonia? No! Mov Disord 2001;16:13-22.

9. Defazio G, Jankovic J. Does peripheral injury induce dystonia? Moving Along. Milwaukee, Wl: Movement Disorders Society, 2010;14:1-9.

10. Fletcher NA, Harding AE, Marsden CD. The relationship between trauma and idiopathic torsion dystonia. J Neurol Neurosurg Psychiatry 1991;54:713-17.

11. Schrag A, Trimble M, Quinn N, et al. The syndrome of fixed dystonia: an evaluation of 103 patients. Brain 2004;127:2360-72.

12. van Hilten JJ, Geraedts EJ, Marinus J. Peripheral trauma and movement disorders Parkinsonism Relat Disord 2007;13(Suppl 3):S395-9.

13. Jankovic J. Post-traumatic movement disorders: central and peripheral mechanisms. Neurology 1994;44:2006-14.

14. Defazio G, Berardelli A, Abbruzzese G, et al. Possible risk factors for primary adult onset dystonia: a case-control investigation by the Italian Movement Disorders Study Group. J Neurol Neurosurg Psychiatry 1998;64:25-32.

15. Roze E, Soumare A, Pironneau I, et al. Case-control study of writer's cramp. Brain 2009;132:756-64.

16. Gioltzoglou T, Milonas I, Lees AJ. Case of DYT1 dystonia triggered by bite from a moray. Mov Disord 2006;21:1536-7.

17. Albanese A, Bentivoglio AR, Del GN, et al. Phenotype variability of dystonia in monozygotic twins. J Neurol 2000;247:148-50.

18. Edwards M, Wood N, Bhatia K. Unusual phenotypes in DYT1 dystonia: a report of five cases and a review of the literature. Mov Disord 2003;18:706-11.

19. Brashear A, Dobyns WB, de Carvalho AP, et al. The phenotypic spectrum of rapidonset dystonia-parkinsonism (RDP) and mutations in the ATP1A3 gene. Brain 2007; 130:828-35.

20. Ibrahim NM, Martino D, van de Warrenburg BP, et al. The prognosis of fixed dystonia: a follow-up study. Parkinsonism Relat Disord 2009;15:592-7.

21. Stone J, Carson A, Aditya $\mathrm{H}$, et al. The role of physical injury in motor and sensory conversion symptoms: a systematic and narrative review. J Psychosom Res 2009;66:383-90.

22. Sa DS, Mailis-Gagnon A, Nicholson $K$, et al. Posttraumatic painful torticollis. Mov Disord 2003;18:1482-91.

23. Schrag A. Psychogenic dystonia and reflex sympathetic dystrophy. In: Hallet M, Fahn S, Jankovic J, et al, eds. Psychogenic movement disorders: neurology and neuropsychiatry. Philadelphia: Lipppincot, Williams and Wilkins, 2006.

24. Scarano VR, Jankovic J. Post-traumatic movement disorders: effect of the legal system on outcome. J Forensic Sci 1998;43:334-9.

25. Thomas M, Jankovic J. Psychogenic movement disorders. In: Shapira A, Lang A, Fahn S, eds. Movement disorders 4. Amsterdam: Saunders Elsevier, 2010.

26. Hallett M. Physiology of psychogenic movement disorders. J Clin Neurosci 2010;17:959-65.

27. van der Salm SM, Koelman JH, Henneke S, et al. Axial jerks: a clinical spectrum ranging from propriospinal to psychogenic myoclonus. J Neurol 2010;257:1349-55.

28. Esposito $\mathbf{M}$, Edwards MJ, Bhatia KP, et al. Idiopathic spinal myoclonus: a clinical and neurophysiological assessment of a movement disorder of uncertain origin. Mov Disord 2009;24:2344-9. 
29. Avanzino L, Martino D, van de Warrenburg BP, et al. Cortical excitability is abnormal in patients with the "fixed dystonia" syndrome. Mov Disord 2008;23:646-52.

30. Espay AJ, Morgante F, Purzner J, et al. Cortical and spinal abnormalities in psychogenic dystonia. Ann Neurol 2006;59:825-34.

31. Quartarone A, Rizzo V, Terranova C, et al. Abnormal sensorimotor plasticity in organic but not in psychogenic dystonia. Brain 2009;132:2871-7.

32. Rosenkranz K. Plasticity and intracortical inhibition in dystonia-methodological reconsiderations. Brain 2010;133:e146.

33. Quartarone A, Rothwell J. Reply: plasticity and intracortical inhibition in dystonia-methodological reconsiderations. Brain 2010;133:e147.

34. Naraghi R, Tanrikulu L, Troescher-Weber R, et al. Classification of neurovascular compression in typical hemifacial spasm: three-dimensional visualization of the facial and the vestibulocochlear nerves. J Neurosurg 2007:107:1154-63.

35. Tsuda M, Inoue K, Salter MW. Neuropathic pain and spinal microglia: a big problem from molecules in "small" glia. Trends Neurosci 2005;28:101-7.

36. Navarro X, Vivo M, Valero-Cabre A. Neural plasticity after peripheral nerve injury and regeneration. Prog Neurobiol 2007;82:163-201.

37. Siegel SM, Lee JW, Oaklander AL. Needlestick distal nerve injury in rats models symptoms of complex regional pain syndrome. Anesth Analg 2007; 105:1820-9.

38. Oaklander AL, Fields HL. Is reflex sympathetic dystrophy/complex regional pain syndrome type I a small-fiber neuropathy? Ann Neurol 2009;65:629-38.
39. Munts AG, van Rijn MA, Geraedts EJ, et al. Thermal hypesthesia in patients with complex regional pain syndrome related dystonia. J Neural Transm

2011;118:599-603.

40. Woolf CJ. Generation of acute pain: central mechanisms. Br Med Bull 1991;47:523-33

41. Saito K, Konishi S, Otsuka M. Antagonism between Lioresal and substance $\mathrm{P}$ in rat spinal cord. Brain Res 1975;97:177-80.

42. van de Beek WJ, Vein A, Hilgevoord AA, et al. Neurophysiologic aspects of patients with generalized or multifocal tonic dystonia of reflex sympathetic dystrophy. J Clin Neurophysiol 2002;19:77-83.

43. Schwenkreis $\mathbf{P}$, Janssen $\mathrm{F}$, Rommel 0 , et al. Bilateral motor cortex disinhibition in complex regional pain syndrome (CRPS) type I of the hand. Neurology 2003;61:515-19.

44. van Hilten BJ, van de Beek WJ, Hoff Jl, et al. Intrathecal baclofen for the treatment of dystonia in patients with reflex sympathetic dystrophy. $N$ Engl J Med 2000:343:625-30.

45. Tinazzi M, Fiorio M, Fiaschi $A$, et al. Sensory functions in dystonia: insights from behavioral studies. Mov Disord 2009;24:1427-36.

46. Quartarone A, Siebner HR, Rothwell JC. Task-specific hand dystonia: can too much plasticity be bad for you? Trends Neurosci 2006;29:192-9.

47. Jankovic J. Medical therapy and botulinum toxin in dystonia. Adv Neurol 1998;78:169-83.

48. Brin MF, Comella CL, Jankovic J, et al. Long-term treatment with botulinum toxin type $\mathrm{A}$ in cervical dystonia has low immunogenicity by mouse protection assay. Mov Disord 2008;23:1353-60.

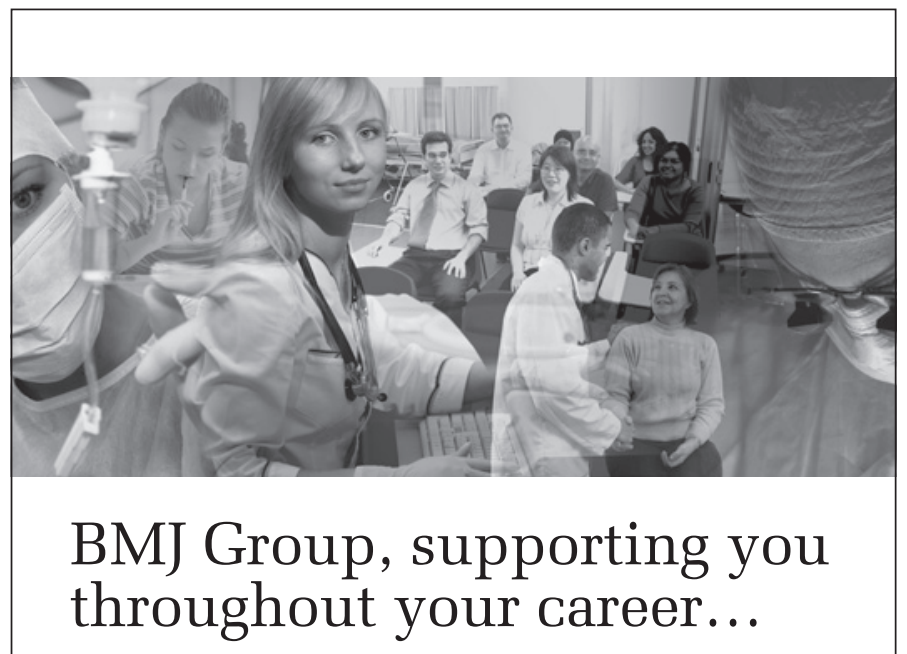

At BMJ Group we have resources available to you at every stage of your career.

Whether you are a medical student or doctor in training looking to keep up with the latest news and prepare for exams, or a qualified doctor who wants the latest medical information, to attend conferences, or looking for your next job, BM] Group has something to offer. For the latest information on all of our products and services register to receive email updates at

group.bmj.com/registration

BMJ BM] BMJIJournals BMJICareers BMJIEvidence Centre BMJILearning (BMyjometa 